\title{
INDUCCIÓN DE LA OVULACIÓN EN LLAMAS MEDIANTE LA ADMINISTRACIÓN INTRAMUSCULAR DEL PLASMA SEMINAL DE LLAMA, ALPACA Y TORO
}

\author{
Alicia López F. ${ }^{1}$, Wilfredo Huanca L. ${ }^{2,3}$, Víctor Leyva V. ${ }^{2}$, Néstor Falcón P.4, \\ Teodosio Huanca M. ${ }^{5}$ y Marcelo Ratto F. ${ }^{6}$
}

\section{Abstract}

Female llama without calf at foot, in good reproductive conditions and bearing a follicle $\geq 7 \mathrm{~mm}$ in the ovaries, detected by ultrasonography, were selected $(\mathrm{n}=64)$. All animals were administered $5 \mathrm{mg}$ of $\mathrm{LH}$ (Lutropin $\mathrm{V}$ ) to synchronize the newly emergent follicular wave. A second ultrasonography evaluation was done 12 days later to confirm the presence of a dominant follicle $(\geq 7 \mathrm{~mm})$ and then, animals were randomly distributed into four groups. The first three groups were i.m. injected $(2 \mathrm{ml})$ with llama, alpaca and bull seminal plasma, respectively, and the fourth group was injected with PBS (phosphate basic serum) and remained as control. The occurrence of ovulation was evaluated two days later. All animals injected with llama and alpaca seminal plasma ovulated, whereas only $25 \%$ of llamas treated with bull seminal plasma, and none of the control group ovulated. The size of the corpus luteum 9 days after treatment did not vary among the treated groups. The results indicated that the i.m. administration of llama and alpaca seminal plasma, and in lesser degree, the bull seminal plasma induce ovulation in the llama and allow the growth of a corpus luteum to a size proper of these species.

Key words: llama, alpaca, bull, seminal plasma, ovulation, corpus luteum

\section{RESUMien}

Se seleccionaron 64 llamas hembras sin cría al pie y en condiciones reproductivas óptimas. El criterio de selección se basó en la presencia de un folículo dominante $\geq 7 \mathrm{~mm}$, detectado por ecografía transrectal. A las hembras seleccionadas se les aplicó $5 \mathrm{mg}$ de LH (Lutropin V) para realizar una sincronización de la emergencia de una nueva onda folicular, confirmándose 12 días después la presencia de un nuevo folículo dominante $(\geq 7 \mathrm{~mm})$. Los animales fueron entonces distribuidos al azar en cuatro grupos. A los primeros tres grupos se les inyectó $2 \mathrm{ml}$ vía i.m. de plasma seminal de llama, alpaca y toro, y al cuarto grupo (control) se le aplicó PBS (suero básico fosfatado). La ocurrencia de ovulación se

\footnotetext{
${ }^{1}$ Práctica privada

${ }^{2}$ Laboratorio de Reproducción Animal, FMV-UNMSM

${ }^{3}$ E-mail: wilfredo.huanca@gmail.com

${ }^{4}$ Laboratorio de Medicina Veterinaria Preventiva, FMV-UNMSM

${ }^{5}$ Instituto Nacional de Investigación Agraria (INIA)

${ }^{6}$ Universidad de Saskatchewan, Canadá
} 
evaluó dos días después, encontrándose que las tratadas con plasma seminal de llamas y alpacas tuvieron una tasa de ovulación del $100 \%$, las tratadas con plasma seminal de toro de $25 \%$ y ninguna en el grupo control. Al noveno día post tratamiento se midió el tamaño del cuerpo lúteo, encontrándose que no existe diferencia significativa entre los grupos tratados. En base a los resultados se puede señalar que la administración intramuscular de plasma seminal de llama, alpaca y en menor grado de toro, induce la ovulación en las llamas hembras y favorece la formación de un cuerpo lúteo de características propias de la especie.

Palabras clave: llama, alpaca, toro, plasma seminal, ovulación, cuerpo lúteo

\section{INTRODUCCIÓN}

La fisiología reproductiva de los camélidos en general difiere de otros animales domésticos por la característica de ser una especie de ovulación inducida; lo que involucra que animales con este tipo de ovulación presenten una serie de mecanismos complejos relacionados con la cópula y el comportamiento de la especie que hasta la fecha no han sido totalmente esclarecidos.

Chen et al. (1985) reportan la ocurrencia de ovulación en camellos bactrianos al depositar semen del camello en la vagina en ausencia de la cópula. Igualmente, Ríos (1989) menciona similares resultados en camélidos sudamericanos y sugiere la presencia de un factor presente en el plasma seminal de alpacas que es capaz de inducir la ovulación. Sin embargo, no existen estudios concluyentes que reporten la inducción de la ovulación con plasma seminal de camélidos sudamericanos.

El presente trabajo se realizó con el propósito de determinar la existencia de un factor o componente bioquímico involucrado en la inducción de la ovulación en el plasma seminal de los camélidos y bovinos. Igualmente, se evaluó el desarrollo del cuerpo lúteo posterior a la ovulación para determinar que la aplicación del plasma seminal no solo induce la ovulación sino tiene capacidad de desarrollar un cuerpo lúteo saludable que facilita la supervivencia embrionaria.

\section{Materiales y Métodos}

Se seleccionaron sesenta y cuatro (64) llamas hembras sin cría al pie y que se encontraban en condiciones reproductivas óptimas. El criterio de selección fue basado en la presenciadeunfolículo dominante $\geq 7 \mathrm{~mm}$, detectado por ecografía transrectal. Para este propósito se utilizó un ecógrafo Aloka SSD 500 y un transductor lineal modo B de 7.5 $\mathrm{MHz}$.

El efecto de diferentes estadios del desarrollo folicular en los animales se eliminó al realizar una sincronización de la emergencia de la onda folicular aplicando $5 \mathrm{mg}$ de $\mathrm{LH}$ (Lutropin V) vía intramuscular.

Los animales se volvieron a evaluar por ecografía 12 días posteriores a la sincronización de la emergencia de la onda folicular para confirmar la presencia de un nuevo folículo dominante $\geq 7 \mathrm{~mm}$, y se distribuyeron al azar en cada uno de los siguientes tratamientos:

- $\mathrm{G}_{1}(\mathrm{n}=16)$ : Plasma seminal de llamas (PS11)

- $\mathrm{G}_{2}(\mathrm{n}=16)$ : Plasma seminal de alpaca (PSa)

- $\mathrm{G}_{3}(\mathrm{n}=16)$ : Plasma seminal de toro (PSt)

- $\mathrm{G}_{4}(\mathrm{n}=16)$ : Suerofosfatado básico (PBS) (grupo control)

Se inyectó $2 \mathrm{ml}$ por vía intramuscular de plasma seminal o PBS a cada animal. 
Cuadro 1. Tasa de ovulación en llamas con folículos $\geq 7 \mathrm{~mm}$ como respuesta a la aplicación intramuscular de PBS y plasma seminal de llama, alpaca y toro

\begin{tabular}{lcccc}
\hline \multirow{2}{*}{ Descripción } & \multicolumn{4}{c}{ Tratamiento $^{1}$} \\
\cline { 2 - 5 } & PBS & PSll & PSa & PSt \\
\hline Animales tratados & 16 & 16 & 16 & 16 \\
Animales que ovularon & 0 & 16 & 16 & 4 \\
$\%$ de ovulación & 0 & 100.0 & 100.0 & 25.0 \\
\hline
\end{tabular}

${ }^{1}$ PBS: Suero fosfatado básico; PSII: Plasma seminal de llama; PSa: Plasma seminal de alpaca; PSt: Plasma seminal de toro

Cuadro 2. Tamaño del cuerpo lúteo $(\mathrm{mm})$ al noveno día de la aplicación intramuscular de plasma seminal en llamas

\begin{tabular}{ccccc}
\hline \multirow{2}{*}{ Tratamiento } & Animales & $\begin{array}{c}\text { C.L. } \\
(\mathrm{mm})\end{array}$ & Límite inferior & Límite Superior \\
\cline { 4 - 5 } & & $10.1 \pm 1.5$ & 9.4 & 10.8 \\
PSII & 16 & $9.8 \pm 2.6$ & 8.5 & 11.0 \\
PSa & 16 & $10.3 \pm 1.3$ & 9.4 & 11.2 \\
PSt & 4 & & & \\
\hline
\end{tabular}

${ }^{1}$ PBS: Suero fosfatado básico; PSII: Plasma seminal de llama; PSa: Plasma seminal de alpaca; PSt: Plasma seminal de toro

Se hicieron evaluaciones ecográficas el día de la aplicación del tratamiento, considerado como día cero $\left(\mathrm{D}_{0}\right)$. La segunda ecografía se realizó 48 horas después con la finalidad de determinar la ocurrencia de ovulación (desaparición del folículo dominante). A los nueve días posteriores al $\mathrm{D}_{0}$ se hizo otra ecografía para detectar la presencia y tamaño del cuerpo lúteo.

\section{Resultados}

El Cuadro 1 muestra la frecuencia de ovulación de cada grupo de trabajo. No hubo ovulaciones espontáneas en el grupo control.
No se encontró diferencia significativa en el tamaño de cuerpo lúteo entre los grupos de animales inyectados con plasma seminal (Cuadro 2).

\section{Discusión}

La tasa de ovulación en los animales a los que se aplicó plasma seminal de llamas y alpacas fue del $100 \%$, en tanto que en aquellos animales tratados con plasma seminal de toro fue de $25 \%$. Estos resultados sugieren la existencia en el plasma seminal de la alpaca, la llama y en menor grado en el toro, de uno o más factores que inducen la ovulación en la llama, coincidiendo con lo reportado en 
camellos bactrianos por Chen et al.(1985) y en alpacas por Ríos (1989). Las diferencias en la tasa de ovulación podrían deberse a la menor concentración de estos factores en el plasma seminal de los bovinos, posiblemente por la presencia de la vesícula seminal presente en esta especie. Cabe la posibilidad que este factor no se forme en esta glándula, que algún componente secretado por ésta bloquee su efecto, o se deba a las características físicas propias del plasma seminal de los bovinos.

Según Pan et al. (2001), este factor es común en camellos bactrianos y en el toro, y su composición química difiere de la forma nativa de LHRH, LH, HCG, PMSG y PGF ${ }_{2 \propto}$, y es semejante a la actividad del GnRH, el cual estimula la liberación de LH y FSH de la pituitaria induciendo la ovulación.

El tamaño del cuerpo lúteo se midió a los 9 días post tratamiento, considerando que el máximo desarrollo se produce en dicho momento (Adams et al., 1991). La falta de diferencia significativa entre grupos sugiere que el factor que induce la ovulación tiene características similares entre las especies.

El 5 al $42 \%$ de las alpacas puede presentar ovulaciones espontáneas sin necesidad de cópula, dependiendo de la estación sexual y la frecuencia de exposición de las hembras a los machos (Fernández Baca et al., 1970; Leyva y Sumar, 1981). También se ha observado ovulaciones sin necesidad de cópula en llamas y que se presentan, al igual que en la alpaca, en el apogeo de la estación sexual (England et al., 1969). En el presente trabajo no se reportó ovulaciones espontáneas en el grupo testigo, lo cual podría explicarse por la ausencia completa de los machos, debido a que su sola presencia genera ciertos estímulos sensitivos, lográndose de esta forma estimular la ovulación (Fernández Baca et al., 1970). Tampoco se observó el efecto hembra que se aprecia en otras especies como la coneja (Staples, 1967).
Los resultados obtenidos en el presente trabajo plantean nuevas hipótesis e interrogantes que deberán ser resueltas a través de observaciones de campo y nuevos estudios a fin de encontrar su fuente de origen y su naturaleza química.

\section{Conclusiones}

- El plasma seminal de la llama y la alpaca contiene uno o varios factores capaces de inducir la ovulación en el 100\% de las llamas al ser aplicado vía intramuscular.

- Este factor también parece estar presente en el plasma seminal del toro, pero en menor concentración o con características químicas diferentes, ya que sólo induce la ovulación en el $25 \%$ de las llamas.

- Este factor sería capaz de favorecer la formación de un cuerpo lúteo fisiológicamente normal.

\section{Literatura Citada}

1. Adams, G.; J. Sumar; O. Ginther. 1991. Form and function of the corpus luteum in llamas. Anim. Reprod. Sci. 24: 127-138.

2. Chen, B.; Z. Yuen; G Pan. 1985. Semen induced ovulation in the Bactrian camel (Camelus bactrianus). J. Reprod. Fertil. 74: 335-339.

3. England, B.; W. Foote; D. Matthews; A. Cardozo; G. Riera. 1969. Ovulation and corpus luteum function in the llama (Lama glama). J. Endocr. 45: 505-513.

4. Fernández Baca, S.; D. Madden; C. Novoa. 1970. Effect of different mating stimulation induction of ovulation in the alpaca. J. Reprod. Fert. 22: 261-267.

5. Leyva, V.; J. Sumar. 1981. Evaluación del peso corporal al empadre sobre la capacidad reproductiva de hembras 
alpacas de un año de edad. IV Conv. Internacional Camélidos Sudamericanos. Punta Arenas, Chile.

6. Pan, G; Z. Chen; X. Liu; D. Li; Q. Xie; F. Ling; L. Fang. 2001. Isolation and purification of the ovulation-inducing factor from seminal plasma in the bactrian camel (Camelus bactrianus). Theriogenology 55: 1863-1879.
7. Ríos, M. 1989. Presencia de un factor de inducción de la ovulación en el semen de alpacas y toro. Tesis de Médico Veterinario. Facultad de Medicina Veterinaria, Univ. Nacional Mayor de San Marcos. Lima. 30 p.

8. Staples, R.E. 1967. Behavioural induction of ovulation in the oestrous rabbit. J. Reprod. Fertil. 13: 429-435. 